\title{
Potential colors for studying stellar populations
}

\author{
Zhongmu Li ${ }^{1},{ }^{2}$ Zhanwen Han $^{1}$ and Fenghui Zhang ${ }^{1}$ \\ ${ }^{1}$ National Astronomical Observatories/Yunnan Observatory, the Chinese Academy \\ of Sciences, Kunming, 650011, China \\ ${ }^{2}$ Graduate School of the Chinese Academy of Sciences \\ email: zhongmu.li@gmail.com
}

\begin{abstract}
We try to find some colors that have the potential to study stellar age and metallicity from $U B V R I J H K$ and ugriz colors using the simple population synthesis model of Bruzual \& Charlot 2003 (BC03). The principal component analysis (PCA) (See Kong \& Cheng 2001) and relative sensitive parameter techniques (see Worthey 1994) are used in this work. As a result, some colors such as $(B-K),(R-K),(I-H),(B-V),(U-R),(R-I)$, are found to have the ability to break the stellar age-metallicity degeneracy partially and can possibly be used for studying stellar populations of galaxies.
\end{abstract}

Keywords. galaxies: photometry, galaxies: stellar content, galaxies: elliptical and lenticular, cD

\section{Introduction}

Determination of ages and metallicities of various stellar populations has long been a very important subject in astronomy and astrophysics, because it can help us to understand not only galaxies but also cosmology. The technique of stellar population synthesis is a powerful tool for such works. However, people usually use spectra-like methods, e.g., absorption line indices, to determine age and metallicity, as colors were shown to be incapable to break the well-known age-metallicity degeneracy. If colors can be used for such works, we can understand stellar populations of galaxies much better, because colors are easier to get than spectra and are independent of the distances of objects. We analyzed the $U B V R I J H K$ and ugriz colors of the standard model of BC03, with an aim of finding some colors that have the potential to break the degeneracy between stellar age and metallicity.

\section{Main results}

1)It is found that $(U-K),(U-H),(U-J),(B-K),(B-H),(U-I),(B-J)$ and $(V-K)$ are more important to determine stellar age and metallicity than other colors, which are independent of the resolution of the population synthesis model and can be used to supervise the multi-color methods of the investigation of stellar populations.

2)Our results show that olors such as $(B-K),(R-K),(I-H)$ are more sensitive to stellar metallicity, while colors such as $(B-V),(U-R),(R-I)$ are more senstive to stellar age than other colors. The detailed metallicity-sensitivities of these colors can be seen in Table 1.

3)Some pairs of colors are shown to have the potential to separate the effects of stellar age and metallicity by our results. For example, $(U-K)$ and $(R-I)$ are more advantageous to study stellar age and metallicity via the low-resolution model of BC03, 
Table 1. Average relative metallicity sensitivities of colors. In the table, Z-sens $=\Delta \mathrm{Age} / \Delta Z$, where $\Delta$ Age and $\Delta Z$ are percentage changes of age and metallicity when they lead to $5 \%$ change in a color respectively. Therefore, colors with bigger Z-sens are more sensitive to stellar metallicity while those with less Z-sens more sensitive to stellar age. The left two columns show results for $U B V R I H J K$ and the right two for ugriz colors. 'High' and 'Low' represent high and low resolution models of BC03 respectively.

\begin{tabular}{|c|c|c|c|c|c|c|c|}
\hline & High & & Low & 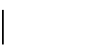 & High & & Low \\
\hline Color & Z-sens & Color & Z-sens & Color & Z-sens & Color & Z-sens \\
\hline B-K & 2.7029 & U-K & 2.7077 & u-z & 1.7542 & u-g & 1.7598 \\
\hline R-K & 2.2928 & I-H & 2.3703 & u-i & 1.5477 & $\mathrm{i}-\mathrm{z}$ & 1.4833 \\
\hline I-H & 2.2870 & R-J & 2.0011 & r-z & 1.5225 & $\mathrm{u}-\mathrm{i}$ & 1.4609 \\
\hline I-J & 2.0531 & R-H & 1.9113 & i-z & 1.4683 & $\mathrm{u}-\mathrm{z}$ & 1.4014 \\
\hline U-K & 2.0250 & R-K & 1.8018 & g-i & 1.4370 & u-r & 1.3825 \\
\hline V-K & 1.9098 & U-R & 1.7760 & g-r & 1.2722 & g-z & 1.1709 \\
\hline R-I & 0.4522 & H-K & 0.5506 & u-r & 1.2659 & g-r & 1.0959 \\
\hline U-R & 0.3974 & $\mathrm{~J}-\mathrm{K}$ & 0.5290 & $\mathrm{r}-\mathrm{i}$ & 1.1425 & g-i & 1.0437 \\
\hline B-V & 0.3695 & R-I & 0.4417 & $u-g$ & 1.0605 & $\mathrm{r}-\mathrm{i}$ & 0.7936 \\
\hline
\end{tabular}

while they should be replaced by $(B-K)$ and $(B-V)$ when using the high-resolution model. Besides, $(u-g)$ and $(r-i)$ seem to have the potential to separate stellar age from metallicity for the low-resolution model of $\mathrm{BC} 03$, but there is no such ugriz colors for the high-resolution model.

4)Models with different resolutions show different pairs of colors for solving the age and metallicity degenerate problem in this study.

5)A few pairs of colors suggested by our results, which seem to have the potential to estimate stellar age and metallicity, are tested in this study.

\section{Disscussion}

Although colors are potential to study stellar populations, the ability of using colors to separate the effects of stellar age and metallicity is not as powerful as line strength indices. If there are ongoing star formations, we will get younger and more metal rich stellar populations than the oldest components of these galaxies, via colors.

\section{Acknowledgements}

This work is supported by the Chinese National Science Foundation (Grant Nos. 10433030, 10521001 and 10303006), and the Chinese Academy of Sciences (No. KJX2SW-T06).

\section{References}

Bruzual, G., \& Charlot, S. 2003, MNRAS, 344, 1000

Kong, X, \& Cheng, F. Z. 2001, MNRAS, 323, 1035

Worthey, G. 1994, ApJS, 95, 107 